\title{
Ungarische Bezüge in der Prager Zeitschrift Ost und West (1837-1848)
}

\author{
MÁRIA RÓZSA
}

H-1113 Budapest, Karolina út 5.

E-mail: rozsam@t-online.hu

(Received: 31 May 2018; accepted: 25 August 2018)

\begin{abstract}
The German cultural periodical Ost und West was published in Prague between 1 July 1837 and 10 June 1848. The goal of its editor, the librarian Rudolf Glaser was the mediation between East and West from Austria, Germany, Russia, and the Balkan countries. A lot of articles were published on Czech, Moravian, Russian, and Polish literature, but the culture of other countries was also mentioned in the form of novels, dramas, poems, travelogues, and other genres about regional matters, music, theatre, etc. Its target readers were the educated bourgeoisie of the age. The literary contributions in Ost und West were submitted by authors. Czech folk poetry played a special role among the subjects. Every now and then, Hungarian authors' works were included (e.g. Pál Jámbor's poem and Károly Kisfaludy's novel) in German translation. Karl Georg Rumy, the enthusiastic collaborator of many German-language papers of the Central European region and a zealous propagator of Hungarian literature sent reports to the journal on several occasions. The reviews of the young Frigyes Szarvady also appeared in Ost und West. Julian Chownitz, among others, reported on local curiosities.

Keywords: German periodical, Prague, connections between Eastern and Western Europe, Hungarian references, Hungarian literature in German translation
\end{abstract}

Die deutschsprachige Kulturzeitschrift Ost und West. Blätter für Kunst, Literatur und geselliges Leben erschien in Prag vom 1. Juli 1837 bis zum 10. Juni 1848. Die Zeitschrift bemühte sich um Vermittlung zwischen Ost und West und enthielt viele Beiträge zur Literatur und Kultur diverser Länder, vor allem zur tschechischen (böhmischen), russischen und polnischen Kultur, aber auch zu anderen Kulturen und Ländern. Mit Novellen auf Fortsetzung, Dramen, Gedichten, Reiseskizzen, Länder- und Völkergemälden, vermischten Aufsätzen über Literatur, Musik, Theater und Kunst versuchte sie den Zielsetzungen einer auf Interesse des Bildungsbürgertums basierenden Zeitschrift nachzukommen. Einige charakteristische Titel seien hier erwähnt: Szenen aus dem Riesengebirge, Der Kurort Karlsbrunn und das mährische Gesenke, Bilder aus der Türkei, Ein Tag an der unteren Save, Bilder aus Ungarn, Die Erhebung des Schneiderhandwerks zur Kunst. Sogar eine Übersicht über die böhmische Literatur vom Jahr 1838 bis Ende 1840 wurde regelmäßig veröffentlicht.

Die Zeitschrift gehörte zum Typ des für den Vormärz charakteristischen deutschsprachigen literarischen Blattes. Reinhard Wittmann beurteilt die Presse dieser Epoche folgendermaßen: „Der vormärzliche Zeitschriftenmarkt war gekennzeichnet durch eine relativ hohe Anzahl belletristischer Journale, die häufig 
Surrogatfunktion für die unterdrückte politische Publizistik besaßen und über ihre unmittelbare ästhetisch-unterhaltende Aufgabe hinaus als Kommunikationsforen des liberalen Bürgertums dienten. [...] Ob im Menzel'schen Literaturblatt oder im Saphirschen Humoristen, ob in Herloßsohns Komet oder den Pfennig- und Hellermagazinen: soweit die Zensur es erlaubte oder übersah, war Politik stets miteinbezogen" (WITTMANN 1982: 149).

Zu diesem Typ gehörten Ludwig August Frankls nach der Revue des Deux Mondes nachgebildete Sonntagsblätter für heimatliche Interessen (1842-1848) in Wien (ZENKER 1900: 26) gleichwie der Pester Spiegel (1829-1852), der 1848 überlebt hatte. Die deutschsprachigen Blätter in Mitteleuropa waren, wenn nicht immer durch persönliche Kontakte, sondern durch für mehrere Organe arbeitende Autoren miteinander vernetzt und übernahmen des Öfteren Nachrichten bzw. Berichte von einander. Die Pest-Ofner deutschsprachigen Zeitungen, die sich zum Teil auf das Material des Prager Ost und West stützend - veröffentlichten eine Vielzahl von Volksliedern und Sagen aus der Folklore der verschiedenen slawischen Völker und berichteten über die slowakischen, böhmischen, slowenischen und serbischen literarisch-kulturellen Bestrebungen (FRIED 1994: 94).

Die in Böhmen und in Ungarn wirkenden deutschsprachigen Autoren hatten sich zum Ziel gesetzt, zwischen der böhmischen bzw. der ungarischen Kultur sowie dem deutschen geistigen Leben zu vermitteln. Dies geschah einerseits durch Übersetzungen aus der jeweiligen Muttersprache ins Deutsche, ,wesentlicher als dies war die Herausbildung einer von der deutschen bzw. österreichischen abweichenden Literatur und Bildung", Presse, Theater und Verlagswesen miteinbegriffen (s. dazu FrIED 1994: 83).

In Prag mit seinen 150.000 Einwohnern gibt es Anfang 184837 unterhaltende Zeitschriften, davon 29 in deutscher Sprache (Hofman 1957: 15-16). Ende Juni 1837 wird ,die neue literarische Erscheinung“ in einem Flugblatt vorangekündigt (Hofman 1957: 27). Ab 1. Juli erschien die Zeitschrift dann regelmäßig dreimal wöchentlich (s. dazu LAISKE 1959: 98). ${ }^{1}$ Ziel der neuen Zeitschrift ist eine literarische Vermittlung zwischen dem slawischen Osten und Deutschland voranzutreiben und somit einen Beitrag zu der Weltliteratur zu geben. Böhmen mit seiner halb slawischen, halb deutschen Bevölkerung, an der Grenze zwischen dem europäischen Osten und dem Westen reich an Literatoren, zeichnete sich dafür aus. Es wurde als „Gegengabe“ auch die Veröffentlichung von deutschen Autoren auch versprochen (HofMAN 1957: 27). Die Thematik des geplanten Blattes reicht von Novellen, Sagen, Märchen, Gedichte, weiterhin Mitteilungen aus dem Gebiete der Philosophie, der Psychologie und Ästhetik ferner der Naturwissenschaften, geschichtliche Darstellungen, Biografien von bedeutenden Literatoren und Künstlern umfassen. Berichte zu Länder- und Völkerkunde bzw. Reiseskizzen, Übersetzungen aus der neueren böhmischen, russischen, polnischen, illyrischen (d. i. krainischen, kroatischen, dalmatinischen, serbischen) Literatur, mit besonderer Berücksichtigung der Volkspoesie werden auch publiziert. Manchen Nummern

\footnotetext{
${ }^{1}$ Für diese Angabe bedanke ich mich bei Frau Pavlina Kafková.
} 
wurden Liedtexte und Noten beigelegt. Daneben erschienen auch Übersichten zu künstlerischen, literarischen und industriellen Bestrebungen in den Hauptstädten Europas. Neben den slawischen Ländern wurde auch Ungarn Beachtung geschenkt. Außerdem wurde über die Leistungen der Prager Bühne regelmäßig informiert. Als Herausgeber wurden Jakob Sambs, ein „einfacher deutscher Postbeamter“, der sein erspartes Kapital dem Zeitungsunternehmen widmete (Hofman 1957: 33) und als Schriftleiter Rudolf Glaser erwähnt (HoFMAN 1957: 28).

Rudolf Glaser (Prag, 1801 - Prag, 1868) war ein früh verwaister Sohn eines Schauspielerehepaares, der Jura und Philosophie studierte. Dann war er als Bibliothekar an der Prager Universitätsbibliothek angestellt und veröffentlichte Gedichte. Glasers Zielsetzung mit seinem Organ Ost und West war, slawische Literatur und Wissenschaft einem deutschsprachigen Publikum näher zu bringen.

Nach der Niederlage der Revolution, in der Ära der politischen Unterdrückung entsagte Glaser jeder publizistischen Tätigkeit und lebte als Skriptor im Klementinum fortan in Zurückgezogenheit bzw. widmete sich dem Studium der orientalischen Sprachen. Er übersetzte „Sakuntala“ aus dem Persischen, diese Übersetzung erschien aber nicht. Ab 1854 verwaltete er die Prager Bibliothek der Fürstenberger und später die des „Vereines für Geschichte der Deutschen in Böhmen“ (HofMAN 1957: 38). Glasers Bedeutung für die böhmische Kulturgeschichte fasst der böhmische Dichter Karl Viktor Hansgirg (Pilsen / Plzeň, Tschechien, 1823 - St. Joachimsthal/Jáchymov, Tschechien, 1877), der ebenfalls regelmäßig in Ost und West publizierte, in seinem Nekrolog folgenderweise zusammen: „Sein Beispiel wird den Historiker belehren, daß der unselige Kampf der Nationalitäten in Böhmen nicht von deutscher Seite begonnen ward. Die Deutschen sind den nationalen Bestrebungen der Čechen friedlich und freundschaftlich entgegengekommen zu einer Zeit, wo die ungeheure Majorität auf ihrer Seite stand und wo es ein Leichtes gewesen wäre, die unbedeutenden Regungen der čechischen Nationalität im Keime zu ersticken" (HANSGIRG 1870: 87).

Die bei Johann Spruny, ab 11. Dezember 1839 bei Karl Geržabek gedruckte, zweispaltig gesetzte Quart-Zeitschrift $(23 \times 30 \mathrm{~cm})$ umfasste mindestens vier-fünf durchnummerierte Seiten.

Der bekannte deutsche Schriftsteller Willibald Alexis (eigentl. Georg Wilhelm Heinrich Häring: Breslau / Wrocław, Polen, 1798 - Arnstadt, Deutschland, 1871), der als Begründer des realistischen historischen Romans in der deutschen Literatur gilt, schickte eine Epistel an den Redakteur. In dieser Epistel stellt er skeptisch seine Meinung über die sehr populären schöngeistigen Blätter fest, deren Urtypen die Zeitung für die elegante Welt, Der Fremüthige für Deutschland, oder das Morgenblatt für gebildete Stände seien. Glasers Vorankündigung bzw. die Zielsetzungen seines neuen Blattes haben aber seine Aufmerksamkeit geweckt, weil Glaser mit dieser Zeitschrift ein Organ der Vermittlung, der Bekanntmachung der slawischen Literaturen geschaffen habe. Dies empfindet er als etwas Neues, noch nicht Dagewesenes. Die slawische Literatur sei nämlich im Westen kaum bekannt, er nennt zwar einige bekannte Autoren wie Aleksandr Puškin (1799-1837), Aleksandr Bestužev-Marlinskij (1797-1839) und den Polen Adam Mickiewicz (1798-1855), 
unter den böhmischen kenne er schon von früher den Prager Karl Egon Ebert (1801-1882) (letzterer publizierte regelmäßig in Ost und West).

Die Zeitschrift war in ganz Europa verbreitet und wurde gelesen (HoFmAN 1957: 55). Ein Beweis für die Zirkulation bzw. Übernahme von Texten in Mitteleuropa ist der Veröffentlichungsweg der Übersetzung des Gedichts „Son“. (,V poldnevnij žar v doline Dagestana...") auf Deutsch: „Der Traum“ („Durchschossen lag in heißer Mittagsstunde...") von Michail Lermontow. Die Übersetzung wurde 1845 in Ost und West mit Angabe des Namens des Übersetzers, [Johann] August Mettlerkamp, Charkow (Hamburg, 1810 - ?, 1859) publiziert. $^{2}$ Im Humoristen erschien ein Nachdruck der Übersetzung am 16. Juli 1846 ohne Angabe des Namens des Übersetzers (Rózsa 2013: 67-68). Der in Wien tätige, aus Ungarn stammende Redakteur des Humoristen, Moritz Gotlieb Saphir mag also die Übersetzung entweder in Ost und West gelesen, oder den gleichfalls 1846 in Braunschweig herausgegebenen Gedichtband „Liederschwalben“ von Mettlerkamp gekannt haben, der diese Übersetzung auch enthielt (REISSNER 1970: 204-205, 362). ${ }^{3}$

Bemerkenswert ist jedoch, dass der Text im Humoristen im Vergleich zu dem in Ost und West die dritte Strophe ausgenommen an mehreren Stellen und zwar zum Besseren korrigiert und fließender gemacht wurde. Das Gedicht ist 1841 entstanden, in dem Jahr, in welchem Lermontow in einem Duell starb. Der verwundete Erzähler liegt in Dagestan auf der Erde, es sind vielleicht bloß Sekunden in seinem Leben übrig und denkt halbwach noch an eine Fest in der Großstadt und an die geliebte Frau. Lermontow kannte den Kaukasus gut, weil er wegen seines Gedichts auf Puškins Tod zu einem Militärregiment dorthin versetzt wurde. Das Gedicht „Der Traum“ ist meisterhaft komponiert: das statische Bild aus der Perspektive des Sterbenden von Unten her ist von zwei Rahmenstrophen umgefasst; einen mehrfachen Kontrast bildet oben die strahlende Sonne in der Wüste, sowie ringsum die Felsen (die Gegenwart) und die heitere Fest mit Kerzenlicht in der Heimat (Vergangenheit).

Das Traummotiv verbindet den Sterbenden mit der geliebten Frau, die einen „düstern Traum“ sieht. Die Übersetzung kann nur gelobt werden, bemerkt werden kann jedoch, dass die Alliteration in der ersten Strophe (,v doline Dagestana“ - im Tal von Dagestan), sowie die Wiederholung des Wortes „,brennen“ und damit die Verstärkung der äußeren und inneren Hitze (,,i solnce žglo... i žglo menja“) und im Gegensatz dazu das kalt werdende Blut des Sterbenden verloren geht. Mettlerkamp war 1840 aus Charkow, wo er seit 1835 als Lektor für Deutsch an der Universität gewirkt hatte, auf Urlaub nach Hamburg gekommen und hier u. a. mit Gutzkow, Hebbel, Dingelstedt und Uffo Horn bekannt geworden. Der ehemalige Offizier in russischen Diensten hatte bereits Puškins kleine Tragödie „Mozart und Salieri“ übersetzt (s. ReISSNER 1970: 204-205). Nach Rußland zurückgekehrt beschäftigte er sich mit der Übersetzung des Lermontow'schen Romans „Der Held

\footnotetext{
${ }^{2}$ Der Traum. Aus dem Russischen des Lermontow. August Mettlerkamp. = Ost und West, № 75, 19. September 1845. 297.

${ }^{3}$ An dieser Stelle bedanke ich mich bei Frau Dr. Anna Ananieva für diese Angabe.
} 
unserer Zeit“" (Sivers 1855: 419-420) aus dem auch Teile 1846 in Ost und West veröffentlicht wurden.

Die Beiträge in Ost und West kamen aus Österreich, Deutschland, Polen, aus den Balkanländern und Rußland. Neben den ständigen Korrespondenten haben deutsche bzw. österreichische Dichter und Schriftsteller wie u. a. Ferdinand Freiligrath, Ludwig August Frankl, Friedrich de la Motte Fouqué, Joseph Mendelssohn, Betty Paoli, Friedrich Rückert, Moritz Gottlieb Saphir, Adolf von Tschabuschnigg, Heinrich Laube, Johann Gabriel Seidl, Karl Beck, Heinrich Ritter von Levitschnigg, Franz Freiherr von Dingelstedt, Karl Gutzkow, Heinrich Wilhelm August Stieglitz, Eduard Gervais, Karl Immermann, Karl Herloßsohn und Johann Nepomuk Vogl mitgewirkt. Unter den tschechisch / böhmischen Autoren veröffentlichten im Blatt u. a. Moritz Hartmann, Sigmund Kolisch, Isidor Heller, Siegfried Kapper, Adolf Neustadt, Uffo Horn, Karl Viktor Hansgirg, Adolf Wiesner und Ludwig Ritter von Rittersberg.

Für Ost und West arbeiteten auch ungarische Korrespondenten. Unter ihnen ist Karl Georg Rumy (Igló / Zipser Neudorf / Spišská Nová Ves, heute: Slowakei, 1780 - Esztergom/Gran, Ungarn, 1847) hervorzuheben; er war ein langjähriger Korrespondent der Zeitschrift. Seine Zeichen waren im Blatt: -u-, m, Dragutin und Karl. Rumy, geboren in der Zips, erlernte die ungarische Sprache erst im reformierten Kollegium zu Debrecen. Danach studierte er Theologie und Naturwissenschaften an deutschen Universitäten. Später unterrichtete er in Lyzeen, dann wurde Professor der Ökonomie zu Keszthely (beim Balaton/Plattensee in Ungarn). $\mathrm{Ab} 1824$ lebte er in Wien, von 1828 an bis zu seinem Tode in Esztergom (Gran). Rumy, der Ökonom, Publizist, Historiker, Übersetzer und Volksliedsammler, mit einem Wort Polyhistor war, war ein Vertreter des sog. hungarus Patriotismus der ungarischen Aufklärung, dessen Tätigkeit - meist auf Deutsch - in den 40er Jahren den Reformbestrebungen der ungarischen Intelligenz schon im Widerspruch stand (FRIED 1963: 204). Er betonte, Jeder sollte frei darüber entscheiden können, welche Sprache er zu Hause, in der Schule oder in der Kirche gebrauchen will (FRIED 1963: 208).

Seine vielseitigen Interessen, seine ständigen finanziellen Sorgen um seine große Familie, sein unruhiges Wanderleben ließen ihm weder Energie noch Zeit zur vertieften Arbeit. Seine geplanten großen Werke hat er nie geschrieben, doch er war bei zahlreichen deutschsprachigen Blättern tätig. Als Publizist erwarb er sich große Verdienste bei der Verbreitung der ungarischen Kultur und korrigierte oft falsche Ansichten über Ungarn. Nach seinem Biographen veröffentlichte er allein im Wiener Wanderer zwischen 1824 und 1835 nicht weniger als 1470 Artikel (KöRÖSY 1880: 94). Er schrieb über die verschiedensten Themen, u. a. über ökonomische Fragen, oder Krankheiten. Rumy berichtete auch über die nationale Unterdrückung der Slowaken durch den nationalistischen magyarischen Adel. Daneben schickte er seine Berichte an fast alle Wiener und Pester Blätter (den Wanderer, die Sonntagsblätter, den Humoristen, das Pesther Tageblatt und den Ungar).

In der Zeitschrift Ost und West meldete er sich zum ersten Mal mit einem Bericht über „Die serbischen Buchdruckereien zu Czettin in der Czerna Gora 
(Montenegro) und zu Belgrad in Serbien" und verbreitete damit Wissenswertes über die Buchkultur der südslawischen Nationalitäten. ${ }^{4}$

Rumy veröffentlichte eine Buchbesprechung über das Buch „Szerb népdalok és hősregék". Az eredetiből fordítá Székács József, kiadá Kunoss Endre [Serbische Volkslieder und Heldensagen. Aus dem Original übersetzt von Joseph Székács, herausgegeben von Andreas Kunoss. Pesth, in der Trattner-Karolyischen Buchdruckerei 1836]. Rumy hält die Übersetzung für gelungen; die Serben besäßen einen Schatz an Volksliedern und Heldensagen, wie sich dessen kein Volk in Europa rühmen könne. Er erwähnt auch den Namen von Vuk Stefanović Karadžić, zum Schluß teilt er eine kleine Probe sowohl in ungarischer als auch in deutscher Sprache aus dem Buch mit. ${ }^{5}$

In deutschsprachigen Periodika Oberungarns vor 1848 wurden die Slowaken tatsächlich „zumeist mit rührseligen und bemitleidenden Worten beschrieben“ stellt Andreas Schriefer in seiner Arbeit über die deutschsprachigen Periodika dieser Region fest. „Diese seien durch vielerlei Faktoren - Beschränkung auf den bäuerlichen Sektor, schlechte Böden, Alkohol usw. - vernachlässigt und in ihrer Entwicklung - zumeist unverschuldet - behindert" (SCHRIEFER 2007: 134). Außerdem herrschte in den 40er Jahren in Oberungarn eine Hungersnot.

Rumy lieferte in einem seiner Artikel philologische Beweise für die slawische und deutsche Herkunft vieler ungarischen Wörter. ${ }^{6}$ In der ersten seiner Äußerungen reagiert Rumy auf einen in der Zeitschrift Hasznos Mulatságok erschienenen Artikel und beantwortet die dort Erschienen mit der Begründung, dass der „Afterpatriotismus", d. i. die Aufzwingung des Ungarischen auf andere Nationalitäten (meist mit slawischen Muttersprachen) schädlich sei. ${ }^{7}$

Rumy verurteilt die Geringschätzung, mit der einige ungarische Zeitschriften von den Slowaken sprechen. So z. B. in der Antwort auf die Bemerkungen des reformierten Predigers Zsigmond Kulifai (1796-1868), erschienen in Tudományos Gyüjtemény (№ 15, 1839) zitiert Rumy Kulifais Feststellungen, dass Tapferkeit, Landwirtschaft, Hospitalität die Hauptneigungen der Magyaren seien. Kulifai schreibt aber nichts über die „dunklen Seiten“ der Magyaren, meint Rumy. Danach nennt er solche Eigenschaften wie Faulenzen, die Verspottung anderer Nationen, die Lauheit gegen die Wissenschaften und ihre Geringschätzung, das feste Ankleben an den alten Schlendrian, das Beneiden der Fortschritte Anderer, den Hochmut, die Ruhmsucht und den Haß der Fremden. ${ }^{8}$

\footnotetext{
${ }^{4}$ Dr. Rumy: Die serbischen Buchdruckereien zu Czettin in der Czerna Gora (Montenegro) und zu Belgrad in Serbien. = Ost und West, № 59, 25. Juni 1838. 244.

${ }_{5}^{5}$ Dr. Rumy: Magyarische Uebersetzung der serbischen Volkslieder. = Ost und West, № 77, 26. September 1838. 318; № 78. 29. September 1838. 321.

${ }^{6}$ Dr. Rumy: Aufrichtiges Geständnis eines magyarischen Gelehrten über den fremden Ursprung vieler magyarischen Wörter, und patriotischer Wunsch. = Ost und West, № 15, 21. Februar 1838. 64.

${ }^{7}$ Ry: Unbefangenes Urtheil eines Magyaren über die Verachtung fremder Sprachen . = Ost und West, № 42, 26. Mai 1838. 174.

${ }^{8}-\mathrm{u}$-: Beitrag zur Schilderung des Nationalcharakters der Magyaren. = Ost und West, № 52, 30. Juni 1838. 216.
} 
Im Aufsatz „Hartes und ungerechtes Urtheil eines Magyaren über die Slowaken Ungarns“, erschienen in der Rubrik „Debatte“ von Ost und West, behandelte Rumy den in der Zeitschrift Hasznos Mulatságok (№ 31, 1837) unter dem Titel „Nemzetek' charactere“ [Charakter der Nationen] erschienenen Artikel des Advokaten Elek Nagy (1820-1875), in dem dieser schreibe, dass die Slowaken ihre Kinder gar nicht erziehen, da sie meistens unwissend und arm seien, lassen sie ihre Kinder wild aufwachsen. Demgegenüber sei Rumy der Meinung, dass der bemittelte und gebildete Slowak seinen Kindern eine so gute Erziehung zu Teil werden lasse, wie der bemittelte und gebildete Deutsche und Magyar in Ungarn. Nagy gehe noch bis zur verurteilenden Verallgemeinerung, dass sie Slowaken neidisch und zum Zorne leicht reizbar, dabei stolz und bis aufs Blut streifsüchtig seien. Rumy ist dagegen der Meinung, dass dies ein ,mißlungenes und ungerechtes Charaktergemälde" sei, weil ,unter den Katholiken und Protestanten Augsburger Confession in Ungarn sehr viele gelehrte Slowaken mit Ruhm Professorstellen bekleideten und noch bekleiden und geschätzte wissenschaftliche Werke herausgaben und herausgeben". Im Weiteren zählt er eine Reihe solcher Wissenschaftler auf. Dass die Slowaken überhaupt neidisch seien, sei eine Verläumdung. Der Magyar sei ebenfalls zum Zorn reizbar, meint er, und im Gegenteil zum Slowaken sei der Magyar stolz, aber auch andere Völker, z. B. die Spanier seien auch stolz. ${ }^{9}$

Der Korrespondent - m - (annehmbar Rumy) reflektiert auf einen Artikel in der ungarischen Zeitschrift Századunk, in dem festgestellt wurde, dass das gelehrte Ausland sich wenig um die Vereinfachung der Buchstaben bekümmere, geht weiterhin auf die seiner Meinung nach richtige Schreibweise einiger ungarischer Wörter ein. Rumy fügt hinzu, dass das Weglassen des Deutschen als Gelehrtensprache in Ungarn seines Erachtens schädlich wäre. ${ }^{10}$

Im „Urteil eines unbefangenen magyarischen Literators über die deutsche Sprache, Journalistik und Literatur in Ungarn“" schließt sich Rumy an die gerechte Rüge Ferdinánd Kapuvárys (?-?) über den gegenwärtigen Zustand des deutschen und ungarischen Theaters in Pest. Zu dieser Zeit wurde das ungarische Nationaltheater schon populärer in Pest als das Deutsche Theater. Er verteidigt das Pesther Tageblatt und betont seine Ansicht, dass die Herausgabe eines deutschsprachigen Blattes keinesfalls Unpatriotismus oder Nationalverbrechen bedeute. Demgegenüber unterstreicht er die Wichtigkeit der deutschen Sprache als "Wehikel“ und Verbreiterin der Kultur. ${ }^{11}$ Rumy verwies auf die Wichtigkeit der kulturellen Vermittlerrolle des Deutschen in deutschsprachigen Periodika u. a. im Pesther Tageblatt mehrmals hin (RózsA 2013: 102).

In einem aus Gran geschickten Korrespondentenbericht nahm Rumy in der Debatte des tschechischen Schriftstellers und Journalisten Karel Sabina (1813-1877)

\footnotetext{
${ }^{9}$ Dr. Rumy: Hartes und ungerechtes Urtheil eines Magyaren über die Slowaken in Ungarn. = Ost und West, № 83, 16. Oktober 1839. 358-359.

${ }^{10}$ - $\mathrm{m}$-: Verschiedenheit des Studiums der Philologie unter den Magyaren und Ausländern. = Ost und West, № 95, 27. November 1839. 415.

${ }^{11}$ Dr. Rumy: Urtheil eines ubefangenen magyarischen Literators über die deutsche Sprache, Journalistik und Literatur in Ungarn. = Ost und West, № 96, 30. November 1839. 422-423.
} 
mit dem Pesther Tageblatt an der Seite des tschechischen Kollegen Stellung, der beanstandete, dass das Blatt der tschechischen Sprache und Literatur ein baldiges Ende vorausgesagt gehabt habe. Im zweiten Teil seiner Schrift reagiert Rumy auf die Schrift eines in Wien lebenden Böhmens, der darüber klagt, dass in Wien in den Kaffeehäusern keine böhmischen/tschechischen Blätter vorhanden seien. Rumy stellt daraufhin fest, dass in Pest im Gegenteil viele Periodika aus mehreren slawischen Sprachen in den Kaffeehäusern zu lesen seien. Er meint, dass daran die Böhmen selber schuld seien, so wie die Ungarn, müssten sie auch selber dafür einiges tun, damit die böhmischen Blätter in den Kaffeehäusern für alle Interessenten erreichbar werden. Rumy gibt übrigens in diesem Schreiben ein Beweis über seine umfassende Kenntnis der verschiedensten südslawischen Journale der Monarchie kund. ${ }^{12}$

In einem Bericht aus Ungarn polemisiert Rumy mit dem Pester Spiegel über den slawischen Ursprung des Helden aus Theodor Körners „Zriny“ und führt Quellen an, die dessen kroatische Abstammung beweisen, berichtet weiterhin über den in Buda herausgegebenen slowenischen literarischen Almanach „Zora“. ${ }^{13}$

„Unter dem, wie immer polemisch zugespitzten Titel 'Controlle des Ultramagyarismus' protestiert Rumy gegen die Magyarisierung der Slowaken in Preßburger Kreisen und beruft sich auf das historische Recht, wonach die Slowaken in ihrer Heimat autochthon seien. Das Verlangen nach einer gemeinsamen Sprache in Ungarn sei unbillig und werde aus politischen Gründen vorgebracht. 'Wahrer Patriotismus läßt sich mit Kosmopolitismus verbinden'..." (zitiert nach HofMAN 1957: 80-81). ${ }^{14}$

Der einem multikulturellen, multiethnischen Gebiet entstammende Rumy konnte vorurteilsfreier in Sachen des Zusammenlebens verschiedener Nationalitäten Stellung nehmen. Seine Hinweise auf die sozialen Umstände der Slowaken und Magyaren basierte auf eigenen Beobachtungen und sind somit als frühe soziologische Bestandsaufnahmen aufzufassen.

Aus Ungarn berichtete Rumy über die Debatte des in Wien lebenden, aus Oberungarn stammenden, mehrsprachigen Advokaten, Dichters, Historikers und Ethnographen Johann Csaplovics / Ján Čaplovič (1780-1847) mit dem Juristen, Schriftsteller, Theaterkritiker und Direktor des Ungarischen Nationaltheaters, Historiker, Politiker und Redakteur, József Bajza (1804-1858) in der Zeitschrift Századunk in Betreff der Magyarisierung des Pesther und Ofner Deutschen Theaters durch das Ungarische Nationaltheater in Pesth. Csaplovics schreibt in Századunk gegen Bajza ironisch, dieser habe die wichtige Entdeckung gemacht, dass das magyarische Nationaltheater in Pesth zu seinem vollen Aufblühen nichts weiter brauche, als Wasser, dass heißt, näher an die Donau versetzt zu werden, weiterhin, dass die Ofner-Pester Deutschen mit der Peitsche ins magyarische Theater getrieben werden müßten. Rumy verteidigt das Recht der deutschsprachigen Einwohner auf

\footnotetext{
${ }^{12}$ Dr. Karl Rumy: Correspondenz aus Gran. = Ost und West, № 96, 30. November 1839. 418419; № 97. 4. Dezember 1893. 427-428.

${ }^{13}$ Dragutin. = Ost und West, № 3, 8. Januar 1840. 11.

${ }^{14}$ S. noch: Karl: Controlle des Ultra-Magyarismus. = Ost und West, № 7, 22. Januar 1840. 27.
} 
ein deutschsprachiges Theater, da unter den 120.000 Einwohnern beider Städte viele Deutsche seien. ${ }^{15}$

In der Rubrik „Notizen“ erschien ein kurzer Bericht über die Etymologie des Wortes „tót“ (Thót) bzw. des Sprichwortes „Tót nem ember“ [Der Slowak sei kein Mensch]. Berufen wird auf den Dramatiker Ivan Kukuljević, der im Wort bei den Vorfahren der Magyaren die Bedeutung „Vater der Weisheit und Halbgott“ entdeckte, demgemäß besäße das Wort keine pejorative Konnotation, sondern eher positive. ${ }^{16}$

Gleichfalls Rumy berichtete über verschiedene Neuigkeiten, u. a. über die Sitzung der Ungarischen Akademie der Wissenschaften am 29. Dezember 1845, an der der Schriftsteller Adolf Frankenburg (1811-1886) seine Antrittsrede hielt. Im Weiteren informierte er über Preisverleihungen der Akademie an ungarische Naturwissenschaftler, über eine geplante literarische Zeitschrift unter dem Titel „Tudomány és Irodalom“, die später nicht verwirklicht wurde. Er erwähnte auch die in Leipzig anonym veröffentlichte Flugschrift „Magyarkák“ (Teubner, 1845) des Advokaten und Redakteurs Mihály Kovacsóczy (1801-1846), in welcher der Verfasser Porträts von ungarischen Schriftstellern zeichnet, die Rumy allerdings nicht gelungen findet und zitiert die Kritik der Zeitung Pesti Hirlap: „Der Verfasser besaß nicht genug Fähigkeit, seiner Aufgabe zu entsprechen... Der Verfasser sagt viel an, hält aber wenig fest“. Schließlich erwähnt Rumy die Schriftstellerin Teréz Karacs (1808-1892), die zu den besseren und beliebteren gehöre und den vom serbischen Professor Peter Jovanović in Neusatz (Újvidék / Novi Sad, heute: Serbien) herausgegebenen Almanach „Bačka Vila“. ${ }^{17}$

In der Rubrik „Literatur“ erschien ein kurzer Bericht über die 1844 bei Heckenast in Pest veröffentlichte deutsche Übersetzung von Gustav Steinacker des Romans von Miklós Jósika (1794-1865) „Zrínyi der Dichter“. ${ }^{18}$ Absicht des Rezensenten war nicht, in die Details des Werkes einzugehen, er erwähnte bloß den reichen Stoff, schilderte den historischen Hintergrund, das in dreigeteilte Ungarn (türkisch besetztes Landesteil, Siebenbürgen in der Zeit des Fürsten Ferenc Rákóczi II. und das habsburgische Landesteil) und die weiblichen Charaktere.

Zwar gehörte die Popularisierung der ungarischen Literatur nicht zu den Hauptaufgaben von Ost und West, es kam doch dreimal zur Veröffentlichung ungarischer belletristischer Werke. 1846 meldete sich Gustav Ignaz Zerffi mit der Übersetzung „Erinnerungsblätter. An eine hochgeborne Dame“ (ung.: „Emléklapok egy förangú hölgyhöz" von Hiador, erschienen 1846 in der Zeitschrift Honderü). ${ }^{19}$ Der Redakteur bemerkt, dass Hiador einer der gefeiersten Dichter Ungarns sei.

${ }^{15} \mathrm{a}-\mathrm{o}$ : Ansicht des Herren Johann v. Csaplovics in Wien in Betreff der Magyarisierung der Pesther und Ofner Deutschen Theater durch das magyarische Nationaltheater in Pesth. = Ost und West, № 31, 16. April 1841. 128.

${ }^{16}$ Thót nem ember. $=$ Ost und West, № 79, 1. Oktober 1841. 320.

${ }_{17}^{17} \mathrm{y}$ : Aus Ungarn. = Ost und West, № 13, 31. Januar 1846. 51.

${ }^{18}$ E.: Zrínyi der Dichter. = Ost und West, № 8, 28. Januar 1845. 31-32.

${ }^{19}$ Erinnerungsblätter. An eine hochgeborne Dame Nach Hiador von G. I. Zerffi. = Ost und West, № 85, 18. Juni 1846. 337; № 88, 25. Juli 1846. 349; № 90, 30. Juli 1846. 357; № 99, 20 August 1846. 393. 
Hiador war der dichterische Name des Gymnasialdirektors, Dichters, Übersetzers und Landtagsabgeordneten Pál Jámbor (Paks, Ungarn, 1821 - Szabadka / Subotica, heute: Serbien, 1897). Jámbor war ein mittelmäßiger Verterer der Biedermeier Almanachlyrik, dessen Dichtung schon von den Zeitgenossen entweder keinen Widerhall bekam oder mit vernichtender Kritik empfangen wurde. Gustav Ignaz Zerffi (geb. Hirsch, Buda / Ofen, Ungarn, 1820 - London, Großbritannien, 1892) war ein Mann mit bewegtem Leben und eine widerspruchsvolle Persönlichkeit, dessen Übersetzungen und Berichte in verschiedenen deutschsprachigen Blättern Ungarns erschienen. Im Vormärz war er Mitarbeiter des Spiegel und am 1. Oktober 1848 übernahm er die Leitung des formal und thematisch umgewandelten Ungar. Die Zeitung Morgenröthe redigierte er vom 1. Januar 1848 bis zum 19. Juli 1848. Zerffi lenkte die Aufmerksamkeit durch Artikel auf sich, in welchen er auf Petőfi schimpfte. 1848 wurde er ein begeisterter Anhänger der Revolution und übersetzte Petôfis „Nationallied“ ins Deutsche (unter dem Titel ,Nationalhymne der Magyaren"). Nach der Niederschlagung des Freiheitskampfes ging er in die Emigration und lebte bis zu seinem Tode in London ein Doppelleben als anerkannter Wissenschaftler und Agent des Wiener Hofes (zu Zerffi s. Frank 2002).

Die romantische Novelle über die moralische Veredelung durch überstandene Proben von Laura, der verwöhnten Ehefrau des Grafen Lerma, deren Schauplatz Madrid ist, übertrug frei aus dem Ungarischen Julie Großmann. Ob die in Schlesien geborene Dichterin Julie Großman (geb. von Menzel, Freistadt / Fryštát, Tschechien, 1790 - Dresden, heute: Deutschland, 1860) tatsächlich ungarisch konnte, oder sie eine Rohübersetzung benutzte, ist nicht mehr feststellbar. Gleichfalls ist der ungarische Autor „S. M.“ nicht identifizierbar. ${ }^{20}$

Manche Berichte über ungarische kulturgeschichtliche Themen stammen im Blatt von Professor Anton[ín] Fähnrich (Št'áhlav u Plzni, Tschechien, 1802 - Jičín, Tschechien, 1852). Fähnrich betätigte sich als Gymnasiallehrer und Doktor der Philosophie bei verschiedenen Blättern, ferner als Herausgeber von tschechischen bzw. deutschsprachigen Wörterbüchern. Er schrieb in Ost und West über „Die geschichtlich erste slawisch christliche Kirche zu Szalavár in Ungarn". ${ }^{21}$ Zalavár [sic!] war eine slawische Siedlung an der Mündung des Flusses Zala in den Plattensee / Balaton in einem Moorgebiet, die Einwohner sind die Slowenen, sie leben bis heute in Ungarn und sprechen wendisch. Die Kirche wurde unter dem slawischen Fürsten Privini erbaut und durch Luitpold, Erzbischof von Salzburg $850 \mathrm{zu}$ Ehren der hl. Maria eingeweiht. Gleichfalls berichtete er über das Badeort Szliács / Sliač im Neusohler Komitat. ${ }^{22}$

${ }^{20}$ Laura. Novelle frei nach dem Ungarischen des S. M. von J[ulie $]$ GroSsmann. = Ost und West, № 27, 4. März 1847. 105-107; № 28, 6. März 1847. 109-110; № 29, 9. März 1847. 113-114; № 30, 11. März 1847. 117-118; № 31, 13. März 1847. 121-122; № 32, 16. März 1847. 125-126; № 33, 18. März 1847. 129-130; № 34, 20. März 1847, 133-134; № 35, 23. März 1847. 137-138.

${ }^{21}$ Ant. Fähnrich: Die geschichtlich erste slawisch christliche Kirche zu Szalavár in Ungarn. = Ost und West, № 67, 5. Juni 1847. 265-267.

${ }^{22}$ Prof. A. Fähnrich: Das elektrische Bad zu Sljać in slaw. Ungarn im Neusohler Comitat. = Ost und West, № 67, 5. Juni 1847. 326. 
Einzig dem sehr schön gedruckten und vom allgemein bekannten Historiker und Dichter, Grafen János Mailáth (1786-1855) herausgegebenen Almanach „Iris“ gelang in dieser Gattung in der gesamten Monarchie anerkannt zu werden. In der Rubrik „Deutsche Literatur“ wurde „Iris“. Deutscher Almanach für das Jahr 1847 von Karl Viktor Hansgirg besprochen. „Wie des Grafen Mailath Jahresgabe durch Adel des Inhalts und Eleganz der Außenhülle vor den Almanachen und Jahrbüchern Süddeutschlands gewöhnlich hervorzuthun pflegt, so auch diesmal“ - beginnt er. Unter den im Band vorhandenen Autoren erwähnt der Rezensent Levitschnigg, Hermann Klein, Stifter und Julie Großmann. ${ }^{23}$

Bloß thematisch ist ein Landschaftsgedicht eines unbekannten Dichters auf Ungarn bezogen, das laut Mitteilung der Redaktion aus Bukarest eingesendet wurde. ${ }^{24}$ Die Erzählung „Was macht der Storch?“ des Dichters, Dramatikers, Erzählers, Redakteurs und eines Organisators des literarischen Lebens im Vormärz, Károly Kisfaludy (Tét, Ungarn, 1788 - Pest, Ungarn, 1830) wurde in drei Fortsetzungen im Jahre 1847 abgedruckt. ${ }^{25}$ Wer der Übersetzer, Adam Süß war, konnte ich nicht herausfinden. In der Novelle wurde im Rahmen einer Anekdote das Problem der feudalen Zurückgebliebenheit im ungarischen Dorf thematisiert. Die Novellen von Kisfaludy sind zwei Haupttypen zuzuordnen, einer davon ist die satirisch-realistische Novelle, in der die zeitgenössische Gesellschaft dargestellt wird, zu welchem auch diese 1824 entstandene Novelle gehört. Der Inhalt der Novelle ist: Bei seiner Durchreise in einem Dorf erfährt der Ich-Erzähler, warum ein toter Storch am Kirchturm hängt und die Geschichte vom Dorfrichter und Dorfnotar, von deren der Letztere den Vogel übrigens völlig sinnlos abgeschossen hatte, vom Dorfzigeuner, der bloß Geige spielen konnte, und der erfolglos versuchte, den Vogel abzuholen, dabei stürzte und den Fuß brach. Bei seinem nächsten Besuch hört er, dass der Vogel schließlich von einem Wirbelwind von der Turm weggeweht wurde. Dies ist ein Beispiel für die Unfähigkeit der Dorfbewohner, übrigens alle typische Gestalten, die die möglichen Unkosten der Herunternahme des Storches von der Turm vermeiden wollten und die so nichts taten. Ihre Schlußfolgerung war zum Schluß, dass die Zeit der beste Arzt der Menschen sei, alle Probleme werden irgendwie mit der Zeit gelöst.

Über lokale Angelegenheiten (kurzgefasste Theaterkritik der Aufführungen der Pester Theater, Konzerte, Neubauten, das vom "gründlichen Gelehrten“ und „geistvollsten, beweglichsten Weltmann", Sigmund Saphir redigierte Pesther Tageblatt) berichtete aus Pest Julian Chownitz (Érsekújvár / Neuhäusel / Nové Zámky, heute: Slowakei, 1814 - Döbling, heute Bezirk von Wien, Österreich, 1888) in seiner Serie „Tags-Noveletten aus Pesth“. Baron Chownitz oder Chowanetz hatte als Sohn eines kaiserlichen Offiziers ein abenteuerliches Leben und war an vielen

${ }^{23}$ Karl Viktor Hansgirg: Iris. Deutscher Almanach für das Jahr 1847. Pest Gustav Heckenast. $=$ Ost und West, № 79, 3. Juli 1847. 315; № 80. 6. Juli 1847. 319 .

${ }^{24}$ St. - b...r: Auf der Ungarnpuszta. = Ost und West, № 82, 10. Juli 1847. 325.

${ }^{25}$ Was macht der Storch? Eine Dorfgeschichte. Frei nach dem Ungarischen des Karl Kisfaludy von Adam Süss. = Ost und West, № 92, 3. August 1847. 365-366; № 93, 5. August 1847. 369-370; № 94, 7. August 1847. 374. 
Orten in Deutschland als Journalist tätig. ${ }^{26}$ Er besuchte die Kadettenschule in Olmütz, diente eine Zeitlang als Offizier, ab 1836 lebte er als Schriftsteller. In seinen in Deutschland veröffentlichten Flugschriften kritisierte er das Metternichsche System, wurde aber für kurze Zeit zum Eisenbahnangestellten in Graz. Nachher betätigte er sich als deutschsprachiger Journalist. 1839 gab er in Pest die Tageszeitung Pesther Charivari heraus, es erschien aber bloß eine einzige Nummer davon, die vom 20. Juni. Er berichtete 1839 im Pesther Tageblatt über Neuigkeiten des gesellschaftlichen Lebens (zu Chownitzs Berichten im Pesther Tageblatt s. RózSA 2013: 174-175). Chownitz finden wir nach der 1848er Märzrevolution wieder in Ungarn; er gab in Pest die radikale Zeitung Opposition (April 1848 Juli 1849) heraus. Der Erfolg seines Blattes, was eigentlich ein Boulevardorgan war, dürfte auch daran liegen, dass er Berichte über Ereignisse vor Ort veröffentlichte, bzw. im Falle von Sensationen und interessanten Begebenheiten auch Sonderdrucke oder Flugschriften herausgab. Das Blatt wurde auch auf der Straße gut verkauft. Chownitz folgte die Ansichten der ungarischen radikalen Politiker, er glaubte aber nicht an den Erfolg des revolutionären Widerstandes. Als Jelačićs Truppen sich näherten, floh er am 25. September 1848 nach Wien, wo er ein Blatt unter demselben Titel gründete. Nach der Wiener Revolution zog er nach Böhmen (zu Chownitz s. Lengyel 1978: 471-491).

Zum ersten Mal berichtete Chownitz über die Aufführungen neuer Originalprodukte im Ungarischen Nationaltheater und erwähnte dabei József Bajzas und Mihály Vörösmartys (1800-1855) Namen. ${ }^{27}$

Chownitz schrieb in seinem zweiteiligen Aufsatz über die Aufführung des Melodrams des Direktors des Pester Deutschen Theaters Alexander Schmid(t) (?-?) „Die Verstoßenen“ und teilte eine kleine Probe aus dem zweiten Akt mit. ${ }^{28}$

Aus Pest berichtete ein Korrespondent mit dem Pseudonym Leuname, er schrieb u. a. über Liszts Auftritt in der ungarischen Hauptstadt. ${ }^{29}$ Aus Igló/Zipser Neudorf/Spišská Nová Ves, heute: Slowakei meldete sich ein gewisser Fr. Waniczek, den ich nicht identifizieren konnte. Er informierte über Musikvereinsnachrichten bzw. über seinen Besuch beim Historien- und Bildnismaler Franz Preiß/ Preyß (Igló / Zipser Neudorf / Spišská Nová Ves, heute: Slowakei, 1797 - Igló / Zipser Neudorf/Spišská Nová Ves, heute: Slowakei, 1842). ${ }^{30}$

Neben anderen ungarischen Städten gab es einen Berichterstatter auch in Pécs / Fünfkirchen, der die Theateraufführungen ferner die Namen der auftretenden

${ }^{26}$ J. F. Chownitz: Tagesnovelletten aus Pesth.= Ost und West, № 59, 25. Juni 1838. 243; № 40, 18. Mai 1839. 164; № 45, 5. Juni 1839. 186; № 60, 27. Juli 1839. 251-252; № 88, 2. November 1839. 382-383; № 94, 23. November 1839. 412.

${ }^{27}$ Chownitz: Mittheilungen aus Pesth. = Ost und West, № 75, 19. September 1838. 309.

${ }^{28}$ J. F. Chownitz: Correspondenz aus Pesth.= Ost und West, № 92, 17. November 1838. 377; № 93, 21. November 1838. 382. Der Titel heißt korrekt: Der Verstoßene, die Uraufführung fand am 22. Oktober 1838 statt (s. dazu: BeLITSKA-SCHOLTZ-SOMORJAi 1995: 6519).

${ }^{29}$ Leuname: Pesther Zustände. = Ost und West, № 10, 1. Februar 1840. 43.

${ }^{30}$ Fr. Waniczek: Brief aus Iglau. = Ost und West, № 51, 25. Juni 1841. 207. 
Schauspieler aufzählte. ${ }^{31}$ Die meistens nicht mehr identifizierbaren ungarischen Korrespondenten vor Ort behandelten ausschließlich lokale Angelegenheiten.

Ein ungarischer Autor im Blatt war der junge Jurastudent Frigyes Szarvady. Der später als Diplomat und Journalist bekannt gewordene Frigyes Szarvady (bis 1843 Hirschl, Újvidék, Neusatz / Újvidék / Novi Sad, heute: Serbien, 1822 - Paris, Frankreich, 1882), war Sohn eines Privatiers. Nach juristischen Studien in Pest, Prag und Wien ließ er sich 1847 vorerst als Rechtsanwalt in Preßburg nieder. In der Folge arbeitete er als Korrespondent u. a. für die Pariser Zeitung Le Constitutionnel und engagierte sich während des ungarischen Freiheitskampfs als Vermittler zwischen Pest und Paris sowie zwischen Pest und Wien im Interesse der Revolution. Nach der Niederschlagung der Revolution ging er zuerst nach Deutschland, wo er 1849 die Broschüre „Auf! Deutschland“ publizierte. Anschließend finden wir ihn als Korrespondenten für die Kölnische Zeitung wieder in Paris, wo er weiter Kontakt mit den ungarischen Emigranten aufrecht hielt. Er war Mitglied des Frankfurter Parlaments, ab 1850 lebte er in England und in Frankreich, kehrte erst nach der 1867er Amnestie zurück und wurde zum Redakteur des Feuilletons der Wiener Neuen Freien Presse (Rózsa 2014). Er versuchte mit seinen in der Zeitung Le National veröffentlichten Artikeln Interesse und Verständnis für die politischen Zustände in Ungarn zu wecken.

In Ost und West veröffentlichte er eine sehr positive Kritik über Julius Mosens (eigtl. Julius Moses, 1803-1867) Drama „Kaiser Otto der Dritte“ (1842). Er nannte es - übrigens übertrieben - „eines der besten Dramen unserer Zeit“ und lobte die konsequente, geniale Darstellung von Charakteren, die gedankenreiche erhabene Diktion, die Durchführung einer welthistorischen Idee, den gerundeten Szenenverlauf, weiterhin die historische Genauigkeit. ${ }^{32}$

Szarvady rezensierte in der Rubrik „Deutsche Literatur“ den 1842er Band des Prager Jahrbuches „Libussa“. Er hob unter den Mitarbeitern des Bandes Uffo Horn, Moritz Hartmann, Friedrich Bach, Karl Egon Ebert, G. J. Kolar und Isidor Heller hervor ${ }^{33}$ Ebenfalls in dieser Rubrik schrieb Szarvady über Theodor Mügges (1802-1861) vierbändigen Roman „Toussaint“. Der vollständige Titel des 1840 in Stuttgart erschienen Romans des deutschen Schriftstellers, der meist Abenteuerromane verfasst hatte, war „Toussaint oder der Negeraufstand in Haiti. Eine historische Erzählung für die Jugend“. Manche Episoden hält Szarvady - wahrscheinlich mit Recht - für ermüdend. ${ }^{34}$ Szarvady berichtete über seine Reise in einem Reisefeuilleton. ${ }^{35}$

${ }^{31}$ L. Shandor: Aus Fünfkirchen. = Ost und West, № 5, 5. Februar 1841. 44.

${ }^{32}$ Friedrich Szarvady: Otto III. Tragödie von Julius Mosen. (Aufgeführt auf der Prager Bühne am 27. April und 3. Mai.) = Ost und West, № 41, 21. Mai 1841. 166; № 42, 25. Mai 1841. 171.

${ }^{33}$ Friedrich Szarvady: Libussa. Jahrbuch für 1842. Herausgegeben von Paul Aloys Klar, $k . k$. Kreiskommissar, Direktor der Versorgungsanstalt für erwachsene Blinde etc. Prag. = Ost und West, № 9, 1. Februar 1842. 35-36.

${ }^{34}$ F. Szarvady: Toussaint. Roman von Theodor Mügge. = Ost und West, № 52, 1. Juli 1842. 209.

${ }^{35}$ Fried. Sz-y.: Badner Briefe. = Ost und West, № 60, 28. Juli 1843. 241-242; № 61, 1. August 1843. 244-245. 
Aus Pest berichtete „Bhd.“ über verschiedene gesellschaftliche Ereignisse, z. B. über Konzerte in der Herminenkapelle im Stadtwäldchen, über Pferderennen, weiterhin über Auftritte einzelner Schauspieler auf den Pester Bühnen. ${ }^{36}$ In seiner nächsten Meldung verriet er seinen Namen. In diesem Bericht informierte er über Herrschaftsankäufe eines Barons, ohne Angabe des Namens, in der Nähe von Pest, über die Erscheinung einer Parodie auf Friedrich Halms „Sohn der Wildniß“ unter dem Titel „Sohn des Waldes“, über Aufführungen sowohl im Pester Deutschen Theater als auch im Ungarischen Nationaltheater und kritisierte den übertriebenen Magyarismus, der den allgemeinen Volksfortschritt hemme. ${ }^{37}$ Wer Bernhard Stolz war, konnte ich nicht herausfinden. Er veröffentlichte im Blatt auch Übersetzungen aus dem Französischen, weiterhin Berichte aus der Walachei.

Aus Ungarn berichtete aufgrund seiner Reiseerlebnisse ein gewisser Ignaz Grünberg und kam zur Feststellung, dass der Nationalcharakter eines Volkes nirgends leichter, als in seinen Vergnügungen zu erkennen ließe. So beschrieb er die Vergnügungen u. a. die Tänze der Dorfjugend in der Nähe von Komorn, am rechten Ufer der Donau. Zwar wurde noch eine Forsetzung versprochen, es erschien aber nichts weiteres. ${ }^{38}$

Ein vermutlich ungarischer Berichterstatter aus Pest und Ofen war Filó, den ich nicht identifizieren konnte. ${ }^{39}$

Ein Berichterstatter aus Preßburg informierte im Plauderton über das Wetter, weiterhin über Theaterklatsch aus dem Deutschen Theater unter dem Direktor Franz /František Pokorny (Lstiboř, Tschechien, 1797 - Wien, Österreich, 1850). ${ }^{40}$ Pokorny war 1822 Klarinettist am Stadttheater in Preßburg/Pozsony/Bratislava, heute: Slowakei, 1827 Orchesterdirektor dieses Theaters, 1829 auch Turmmeister von Preßburg, 1835 übernahm er das verschuldete Stadttheater und führte es, besonders auf dem Gebiet der Oper, zu großen Erfolgen (zu Pokorny s. REITTERER 1980). In seinem nächsten Bericht schrieb L. K. darüber, dass die Vorbereitungen zur Sitzung des ungarischen Landtags im Mai vorgenommen wurden, machte die Leser auf den Artikel über magyarische Journalistik der Zeitschrift Pannonia, redigiert „,vom geistreichen Schriftsteller Adolf Neustadt“, aufmerksam, berichtete über einen Artikel in Századunk, weiterhin über die angebliche Abstammung der Deutschen aus Lappland. ${ }^{41}$

In der Rubrik „Historische Denkwürdigkeiten“" schilderte Fr. F. die Eroberung der Festung Szigetvár 1566, den heldenhaften Kampf der ungarischen Verteidiger und den Tod des Sultans Soliman I. [richtig: Suleiman II.] im Lager nach dem tür-

\footnotetext{
${ }^{36}$ Bhd.: Aus Pesth. = Ost und West, № 50, 24. Juni 1842. 201-202; № 51, 28. Juni 1842. 205 206.

${ }^{37}$ Bernhard Stolz: Pesther Briefe. = Ost und West, № 65, 16. August 1842. 261-262; № 66, 19. August 1842. 265.

${ }^{38}$ Ignaz Grünberg: Bilder aus Ungarn. = Ost und West, № 66, 19. August 1842. 265; № 68, 26. August 1842. 273-274; № 69, 30. August 1842. 277.

${ }^{39}$ Filó: Altes und Heuriges aus Pesth und Ofen. = Ost und West, № 87, 1. November 1842. 349-350.

${ }^{40}$ L. K.: Aus Preßburg. = Ost und West, № 14, 17. Februar 1843. 54-55.

${ }^{41}$ L. K.: Aus Preßburg. = Ost und West, № 17, 28. Februar 1843. 67.
} 
kischen Sieg. Eine Frau kleidete sich als Mann an und focht im Kampf für die Verteidigung der Festung gegen die Türken. Nach der Eroberung der Festung haben die Türken den Kopf Zrínyis und anderer getöteten Verteidiger auf hohe Stangen aufgesetzt und so ihren Sieg gefeiert. Es wird noch Sultan Solimans Tod erzählt, er starb in Fünfkirchen (Pécs) drei Tage vor der Eroberung von Szigetvár an den Folgen eines Schlaganfalls. Sein Nachfolger wurde sein Sohn, Selim II. Im weiteren erfahren wir die Umstände des Begräbnisses von Soliman [Suleiman] in Konstantinopel. ${ }^{42}$

1848 erschien die romantische Novelle vom tschechischen Autor Viktor Bednár (?-?) über das Leben des ungarischen Barons Szilágyi mit unerwarteten Wendungen, Umwandlungen, wunderbaren Heilungen, die den Österreichischen Erbfolgekrieg (1740-1748) und die darauf folgenden Jahrzehnte zum historischen Hintergrund hatte. ${ }^{43}$

Die europäische revolutionäre Welle fing am 12. Januar 1848 in Palermo an, danach folgten Paris, München, Wien, Prag und Pest-Ofen. Im Tschechischen Königtum und in der damit vereinten mährischen Markgrafschaft existierte der Wunsch nach gesellschaftlichen Umwandlungen. Die Einwohner verlangten für sich die Gleichberechtigung mit den Deutschen, selbstverständlich im Rahmen des Habsburgerreichs. Eben deshalb nahmen die tschechischen Abgeordneten am gesamtdeutschen konstituierenden Reichstag in Frankfurt nicht teil. Im März 1848 bejubelte Ost und West den Sieg der Revolution und lud alle Literatoren böhmischer und deutscher Zunge zu einer Zusammenkunft am 18. März ein (HofmaN 1957: 87). Die erste Reaktion auf die „märchenhaften Ereignisse“ in Europa schrieb Rudolf Glaser am 18. März und begrüßte die den Völkern Österreichs verliehene Konstitution. Er zählte die Ereignisse in Prag, die Bürgerversammlung, die Aufforderung zur Unterschrift der Petition und die Bildung der Communalgarde auf. ${ }^{44}$ Über die Pester Revolution am 15. März informierte Ost und West anhand der Pester Zeitung, die bis zur Aufhebung der Zensur die Regierung unterstützte. Es wurde über die Forderungen in den 12 Punkten für die ungarische Nation, die Begründung der Pester Bürger-Miliz, ferner über die Befreiung des Schriftstellers Mihály Táncsics (1799-1884) aus der Haft in Buda berichtet. ${ }^{45}$ Glaser schrieb seine Leitartikel während der kritischen Periode regelmäßig unter dem Titel ,Zeitbetrachtungen“. Am 1. April warnte er in einem Leitartikel vor dem Vorhaben, die neue Verfassung auf der alten böhmischen ständischen Grundlage aufbauen zu wollen (HofmAn 1957: 89). Seiner Meinung nach sollte eine erweiterte Vertretung des Bürgerstandes mit neuen, bloß vom Volke gewählten Vertretern aufgebaut werden. ${ }^{46}$ Für Glasers soziales Gefühl ist charakteristisch, dass er in einem Aufsatz

\footnotetext{
${ }^{42}$ Fr. F.: Merkwürdige That einer Frau bei der Belagerung von Szigeth. = Ost und West, № 22, 21. Februar 1846. 87; № 24, 26. Februar 1846. 95.

${ }^{43}$ Viktor Bednár: Der Ungar. Novelle. = Ost und West, № 25, 26. Februar 1848. 97-99; № 26,

29. Februar 1848. 101-102; № 27, 2. März 1848. 105-107; № 28, 4. März 1848. 109-111.

${ }^{44}$ R. G., Prag 18. März. = Ost und West, № 34, 18. März 1848. 133.

${ }^{45}$ Aus Pest. = Ost und West, № 36, 23. März 1848. 143-144.

${ }^{46}$ Rudolf Glaser: Zeitbetrachtungen. $=$ Ost und West, № 40, 1. April 1848. 157.
} 
die Prager mahnt, nicht zu vergessen, dass durch die Erhebung der Völker nicht nur die Bürger, der Mittelstand freigeworden sei, sondern auch die untersten Schichten der Gesellschaft (Hofman 1957: 90). Am 25. April kommentiert Glaser Pillersdorfs [Franz Freiherr von Pillersdorf (Brünn /Mähren, heute: Tschechien, 1786 - Wien, Österreich, 1862) österreichischer Beamter und Staatsmann, kurzzeitig Minister des Inneren bzw. Ministerpräsident, Verfasser der 1848er liberalen österreichischen Verfassung] Erklärung, dass der Beitritt Österreichs zum Deutschen Bund von der Gewährleistung völliger Selbständigkeit der inneren Verwaltung und in Fragen seiner Sonderinteressen abhänge. Der antiösterreichische Glaser sieht in diesem Standpunkt eine Schwächung der demokratischen Stellung des deutschen Parlaments, ein Aufgeben der revolutionären Errungenschaften (HoFMAN 1957: 95-96). Glasers antiaristokratischer Standpunkt offenbart sich auch in diesem Artikel, er ist dessen bewußt, dass die Aristokratie den Verband mit Deutschland nicht ertragen könne. In Bezug auf Ungarn ist er der Meinung, dass kein Land so große Veränderungen durchmachen werde als Ungarn. Die Macht des Adels und der Geistlichkeit sei schon gebrochen, es bestehe aber noch ein Kampf der Religionen bzw. der Nationalitäten bevor. ${ }^{47}$

Im Frühjahr 1848 gab es tschechischerseits keine Sympathiekundgebungen Ungarn gegenüber, später änderte sich die Situation (PRAŽÁK 2004: 75-76) Glaser als Herausgeber einer kulturpolitischen Zeitschrift beschäftigte selbstverständlich die Verwirklichung der Preßfreiheit und er lehnte alle Zwangsmaßnahmen ab, die im neuen Preßgesetz die Rechte der Schriftsteller beschränkten (Hofman 1957: 100). Am 2. Juni 1848 wurde der Kongress der Slawen in Prag zusammengerufen mit dem Ziel, das Habsburgerreich zu einem föderativen Staat umzuwandeln. Der Aufstand der Studenten und Bürger in Prag unterbrach den Kongress, wurde aber nach ein paar Tagen von Windisch-Graetz niedergeschlagen. Ost und West existierte zu dieser Zeit nicht mehr, die letzte Nummer, die 70., erschien am 10. Juni, obwohl am 3. Juni noch die Pränumerationsanzeige für das nächste Halbjahr abgedruckt wurde.

Die Wiener Behörden beobachteten die internationalen Beziehungen des Herausgebers schon seit langer Zeit mit Mißtrauen und Polizeiminister Sedlnitzky ließ Glasers Tätigkeit insgeheim überwachen (s. dazu Mestan 1986: 208). Dies gehört zu den Ursachen neben der „Nationalisierung“ auf der deutschen und tschechischen Seite, die zur Einstellung der Zeitschrift führten. Dazu kamen noch die wirtschaftlichen Schwierigkeiten, infolge deren Glaser oft nicht Honorare zahlen konnte, weiterhin seine lähmenden Geplänkel mit der Zensur (Hofman 1957: 35). Die Entscheidung über die Einstellung der Zeitschrift mag aber irgendwie unerwartet gekommen sein, Glaser befasste sich in der letzten Nummer mit der Frage der Slawen im Habsburgerreich und hoffte auf eine friedliche Lösung. Der Kaiser habe an alle Generalkommandos den Befehl erlassen, dass sie ausschließlich dem Palatin bzw. dem ungarischen Ministerium gehorchen sollen. ${ }^{48}$

${ }^{47}$ Rud. Glaser: Zeitbetrachtungen . $=$ Ost und West, № 50, 25. April 1848. 197-198.

${ }^{48}$ Rudolph [!] Glaser: Zeitbetrachtungen . = Ost und West, № 70, 10. Juni 1848. 277. 
Die zwölf Bände von Ost und West sind ein Ergebnis der Zusammenarbeit der Mitarbeiter bzw. Berichterstatter der Zeitschrift. Glasers Verdienst bestehe in seiner organisatorischen, verbindenen Tätigkeit in Bezug auf die deutsch-slawischen Beziehungen (HoFmAN 1957: 41). „Einer literarischen Zeitschrift obliegt es, nicht nur durch passive Mitteilung an der Entwicklung des schöngeistigen Schaffens mitzuwirken, sondern auch tatkräftig als kritisches Sprachrohr an der gesamten geistigen Kultur teilzuhaben. Ost und West gelang es oft über die slawischen Literatur in reportagenhaften Zuschnitt zu berichten“"(HofMAN 1957: 48). Ungarische Thematik war aber trotz der grundsätzlich slawischen bzw. deutschen Orientierung des Redakteurs im Blatt ständig präsent. Einen großen Anteil daran hatte der mehrsprachige, multikulturelle Karl Georg Rumy, entschlossener Anhänger der slawischen Sprachen und Literaturen bzw. eifriger Popularisator der ungarischen Kultur in deutschsprachigen Periodika der gesamten mitteleuropäischen Region. Neben ihm war eine Zeit lang Julian Chownitz Korrespondent, der gleichfalls aus dem mehrsprachigen oberungarischen Gebiet stammte. Der junge Friedrich Szarvady veröffentlichte einige Rezensionen im Blatt. Erwähnt werden soll, dass die Mitarbeiter der Pester deutschsprachigen Periodika Ost und West ständig im Auge behielten bzw. auf dessen Inhalt reflektierten. Außer den Genannten berichteten andere, heute nicht mehr identifizierbare Korrespondenten über ungarische literarische, kulturelle und gesellschaftliche Aktualitäten. Glaser gelang mit seinem Blatt ein großes, in Mitteleuropa anerkanntes und die fortschrittlichen Kräfte mindestens virtuell vernetzendes Organ zu schaffen, was den ungarischen deutschsprachigen Blättern der Zeit in diesem Maße nicht gelang.

\section{Literatur}

Belitska-ScholtZ-Somorjai 1995 = Belitska-Scholtz Hedvig, Somorjai Olga (Hrsg.): Deutsche Theater in Pest und Ofen 1770-1850. Bd. 2. Budapest: Argumentum, 1995. FRANK 2002 = FRANK Tibor: Ein Diener seiner Herren. Werdegang des österreichischen Geheimagenten Gustav Zerffi (1820-1892). Wien: Böhlau, 2002.

FRIED 1963 = FRIED István: Rumy Károly György, a kultúraközvetítő, 1828-1847 [Karl Georg Rumy, der Kulturvermittler, 1828-1847]. Filológiai Közlöny 9 (1963): 204-218.

FRIED 1994 = FRIED István: Die Kultur des Bürgertums deutscher Muttersprache im PestOfen zur Zeit des Vormärz. In: Sснwов Anton (Hrsg.): Methodologische und literarhistorische Studien zur deutschen Literatur Ostmittel- und Südosteuropas. München: Südostdeutsches Kulturwerk, 1994. 81-94.

HANSGIRG 1870 = HANSGIRG Karl Viktor: Rudolf Glaser (Nekrolog). In: Mitteilungen des Vereins für Geschichte der Deutschen in Böhmen 8 (1870): 87.

Hofman 1957 = Hofman Alois: Die Prager Zeitschrift „Ost und West": Ein Beitrag zur Geschichte der deutsch-slawischen Verständigung im Vormärz. Berlin: Akademie Verlag, 1957.

KÖRÖSY 1880 = KÖRÖsY László: Rumy élete [Rumys Leben]. Budapest-Esztergom: Aigner-Buzárovits, 1880.

LAISKE 1959 = LAISKE Miroslav (red.): Časopisectví v Čechách 1650-1847: př́spěvek $k$ soupisu periodického tisku, zejména novin a časopisủ. Praha: Státní knihovna ČSR, Národní knihovna, 1959. 
LENGYel 1980 = S. LENGYEL Márta: Egy fejezet 1848-i sajtónk történetéből: Julian Chownitz „Die Opposition”-ja [Ein Kapitel aus der Geschichte unserer Presse in 1848. „Die Opposition“von Julian Chownitz]. In: OSZK Évkönyv 1978. Budapest, 1980. 471-491.

MESTAN 1986 = MeSTAN Antonin: Die Monatsschrift der Gesellschaft des Vaterländischen Museums in Böhmen und Ost und West. In: FrIED István, ROSENSTRAUCH-KÖNIGSBERG Edith, LemBerg Hans (Hrsg.): Zeitschriften und Zeitungen des XVIII. und XIX. Jahrhunderts in Mittel- und Osteuropa. Berlin: Ulrich Camen, 1986. 205-210.

PRAŽÁK 2004 = PRAŽÁK Richard: Az 1848-1849-es magyar forradalom és szabadságharc cseh- és morvaországi visszhangja [Der tschechisch-mährische Widerhall der ungarischen Revolution und des Freiheitskampfes 1848/1849]. Publicationes Univestitatis Miskolciensis. Sectio Philosophica 2004/1: 75-80.

REISSNER 1970 = REISSNER Eberhard: Deutschland und die russische Literatur 1800-1848. Berlin: Akademie Verlag, 1970.

REITTERER 1980 = REITTERER H. Franz Pokorny. In: Österreichisches Biographisches Lexikon. 1815-1950. Bd. 8. (Lfg. 37, 1980). 161f. http://www.biographien.ac.at/oebl.

RózSA 2013 = RózsA Maria: Wiener und Pester Blätter des Vormärz und ihre Rolle an der Kulturvermittlung. Kontakte, Parallelen, Literaturvermittlung, Redakteure und Mitarbeiter. (Studien zur Literaturwissenschaft 6.) Herne: Gabriele Schäfer, 2013.

RózSA 2014 = RózSA Maria: Szarvady, Frigyes. In: Österreichisches Biographisches Lexikon. Online-Edition, Lfg. 3 (15.11.2014). http://www.biographien.ac.at/oebl.

SCHRIEFER 2007 = SCHRIEFER Andreas: Deutsche, Slowaken und Magyaren im Spiegel deutschsprachiger historischer Zeitungen und Zeitschriften in der Slowakei. Komárno: Forum Institute, 2007.

SIVERS 1855 = SIVERS Jegór von: Deutsche Dichter in Russland. Studien zur Literaturgeschichte. Berlin: E. H. Schroeder, 1855.

WiTTMANN 1982 = WitTMANN Reinhard: Buchmarkt und Lektüre im 18. und 19. Jahrhundert. Beiträge zum literarischen Leben 1750-1880. Tübingen: Niemeyer, 1982.

ZENKER 1900 = ZENKER Ernst Victor: Geschichte der Journalistik in Österreich. Wien: Hof- und Staatsdruck, 1900. 